\title{
Erratum to: CXCL14 and MCP1 are potent trophic factors associated with cell migration and angiogenesis leading to higher regenerative potential of dental pulp side population cells
}

\author{
Yuki Hayashi ${ }^{1,2}$, Masashi Murakami ${ }^{1}$, Rei Kawamura ${ }^{1,3}$, Ryo Ishizaka $^{2}$, Osamu Fukuta ${ }^{2}$ and Misako Nakashima ${ }^{1 *}$
}

\section{Erratum}

Following the publication of our article in Stem Cell Research $\mathcal{E}$ Therapy [1], we have become aware that errors were introduced inadvertently in Fig. 2.

During the preparation of panel J, the bands of $\beta$-actin were cut out and attached in the middle to remove nonspecific bands. The bands of all samples were located on the same membrane. Unfortunately this panel was included in our article by mistake.

We are now providing a new version of Fig. 2 below which presents in panel $J$ experimental data which were obtained at the same time.

The protein band intensity was re-quantified by densitometry (CS Analyzer) using the correct TRH-DE and $\beta$ actin band. Relative protein expression level was evaluated on the basis of band intensity of TRH-DE/ $\beta$-actin. Each expression level of normal pulp was defined as 1.0.

We apologize for this error and confirm that the conclusions of the article are not affected.

\section{Author details \\ 'Department of Dental Regenerative Medicine, Center of Advanced Medicine for Dental and Oral Diseases, National Center for Geriatrics and Gerontology, Research Institute, Morioka 7-430, Obu, Aichi 474-8511, Japan. ${ }^{2}$ Department of Pediatric Dentistry, School of Dentistry, Aichi-Gakuin University suemoridouri 2-11, Nagoya, Aichi 464-8651, Japan. ${ }^{3}$ Department of Gerodontology, School of Dentistry, Aichi-Gakuin University suemoridouri 2-11, Nagoya, Aichi 464-8651, Japan.}

Received: 7 June 2016 Revised: 7 June 2016

Accepted: 10 June 2016 Published online: 27 June 2016
References

1. Hayashi Y, Murakami M, Kawamura R, Ishizaka R, Fukuta O, Nakashima M. CXCL14 and MCP1 are potent trophic factors associated with cell migration and angiogenesis leading to higher regenerative potential of dental pulp side population cells. Stem Cell Res Ther. 2015;6:111.

Submit your next manuscript to BioMed Central and we will help you at every step:

- We accept pre-submission inquiries

- Our selector tool helps you to find the most relevant journal

- We provide round the clock customer support

- Convenient online submission

- Thorough peer review

- Inclusion in PubMed and all major indexing services

\footnotetext{
* Correspondence: misako@ncgg.go.jp

'Department of Dental Regenerative Medicine, Center of Advanced Medicine for Dental and Oral Diseases, National Center for Geriatrics and Gerontology, Research Institute, Morioka 7-430, Obu, Aichi 474-8511, Japan
}

\section{- Maximum visibility for your research}

Submit your manuscript at www.biomedcentral.com/submit 

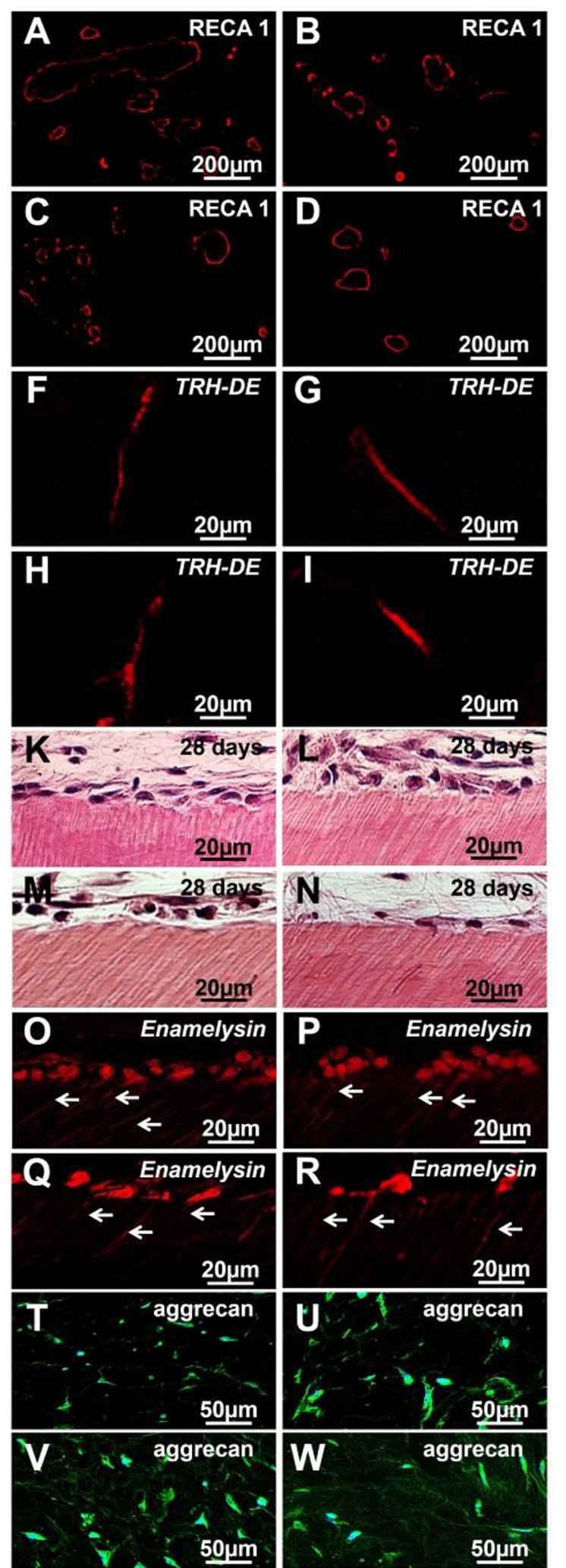

$20 \mu \mathrm{m}$

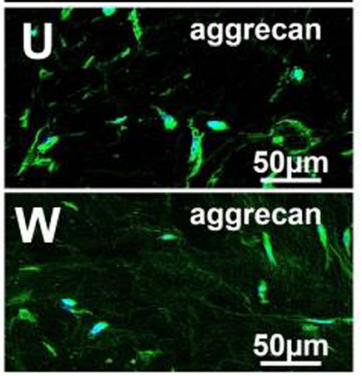

E

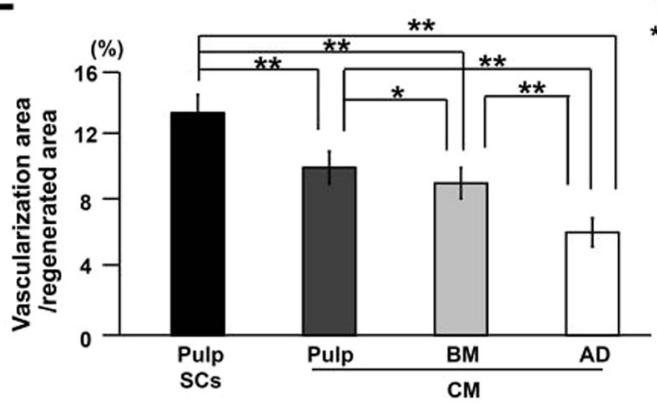

J

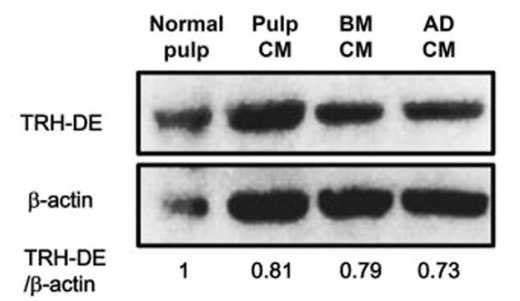

S

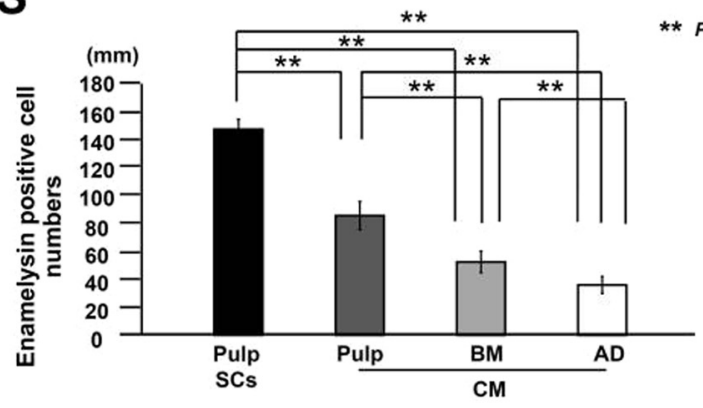

X

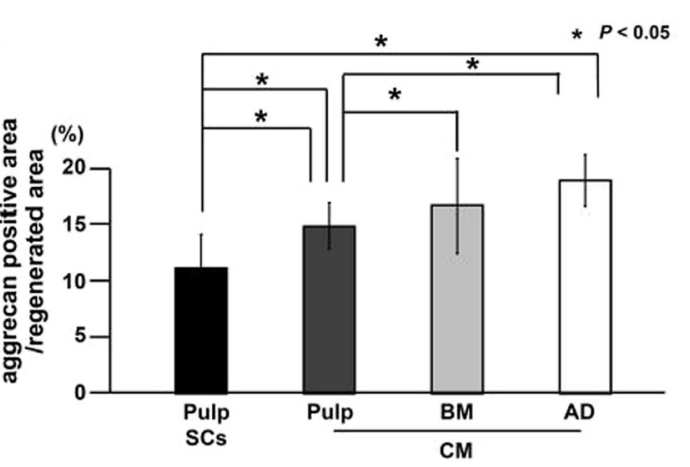

Fig. 2 (See legend on next page.) 
(See figure on previous page.)

Fig. 2 Characterization of regenerated tissue on day 28 in an ectopic tooth root transplantation model. $\mathbf{a}, \mathbf{f}, \mathbf{k}, \mathbf{o}$, $\mathbf{t}$ Transplant of pulp CD31 ${ }^{-}$side population (SP) cells (Pulp SCS). $\mathbf{b}, \mathbf{g}, \mathbf{I}, \mathbf{p}$, u Transplant of conditioned medium (CM) from pulp CD31- SP cells (Pulp CM). $\mathbf{c}, \mathbf{h}, \mathbf{m}, \mathbf{q}, \mathbf{v}$ Transplant of CM from bone marrow CD31- SP cells (BM CM). $\mathbf{d}$, i,n, $\mathbf{r}$, $\mathbf{w}$ Transplant of CM from adipose CD31- SP cells (AD CM). a-d Immunostaining with rat endothelial cell antigen 1 (RECA1). e Ratio of vascularization area to the total regenerated area. (f-i) In situ hybridization analysis of expression of thyrotropin-releasing hormone-degrading enzyme (TRH-DE) as a pulp marker using an anti-sense probe reactive to both porcine and mouse genes. $\mathbf{j}$ Protein expression of TRH-DE in regenerated pulp after transplantation of CM from pulp, bone marrow (BM), and adipose (AD) CD31- SP cells. k-s Odontoblastic differentiation potential in the regenerated pulp. k-n Odontoblastic cells along with the dentinal wall. o-r In situ hybridization analysis of enamelysin. Odontoblastic process extending into the tubular dentin (arrows). $\mathbf{s}$ Comparison of the numbers of enamelysin-positive cells along the dentinal wall. t-w Immunostaining with aggrecan (green) merged with Hoechst 33342 (Blue). x Ratio of aggrecanpositive area to the total regenerated area. Data are expressed as mean \pm standard deviation of four determinations. ${ }^{*} P<0.05$, ${ }^{* *} P<0.01$ 